\title{
Parameters for the injection, acceleration, and extraction of gold and copper ions in Booster, AGS, and RHIC
}

\section{C.J. Gardner}

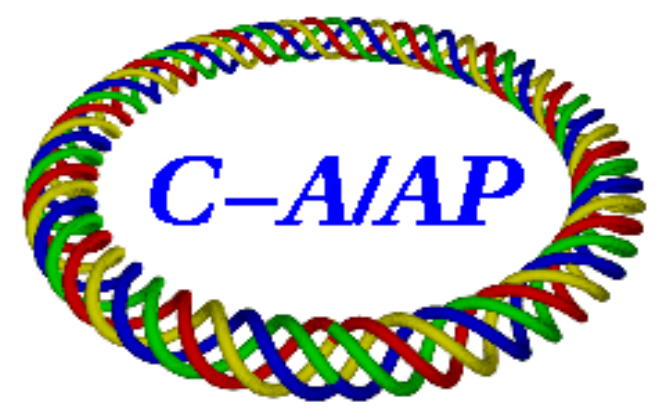

\section{Collider-Accelerator Department Brookhaven National Laboratory Upton, NY 11973}

Notice: This document has been authorized by employees of Brookhaven Science Associates, LLC under Contract No. DE-AC02-98CH10886 with the U.S. Department of Energy. The United States Government retains a nonexclusive, paid-up, irrevocable, world-wide license to publish or reproduce the published form of this document, or allow others to do so, for United States Government purposes. 


\title{
Parameters for the Injection, Acceleration, and Extraction of Gold and Copper Ions in Booster, AGS, and RHIC
}

\author{
C.J. Gardner
}

November 27, 2012

During the 2012 RHIC run, uranium, gold, and copper ions from EBIS were accelerated in Booster and AGS and delivered to RHIC for the physics program. In this note the nominal parameters for gold and copper are given. The parameters for uranium are given in a separate note.

In order to achieve the desired number of ions per bunch in RHIC, it was necessary to merge bunches in both Booster and AGS. This is described in Sections 11 and 12 of Reference [1].

\section{Mass}

A gold ion with charge $e Q$ has $N=118$ neutrons, $Z=79$ protons, and $(Z-Q)$ electrons. Here $Q$ is an integer and $e$ is the positive elementary charge. The mass number is

$$
A=N+Z=197 \text {. }
$$

This is also called the number of nucleons. The mass energy equivalent of the ion is

$$
m c^{2}=a m_{u} c^{2}-Q m_{e} c^{2}+E_{Q}
$$

where $[2,3]$

$$
a=196.9665687(6)
$$

is the relative atomic mass of the neutral gold atom,

$$
m_{u} c^{2}=931.494061(21) \mathrm{MeV}
$$


is the mass energy equivalent of the atomic mass constant, and

$$
m_{e} c^{2}=0.510998928(11) \mathrm{MeV}
$$

is the electron mass energy equivalent. The binding energy $E_{Q}$ is the energy required to remove $Q$ electrons from the neutral gold atom. This amounts to $[4,5] 0.332 \mathrm{MeV}$ for the helium-like gold ion $(Q=77)$ and $0.517 \mathrm{MeV}$ for the fully stripped ion. For $Q=32$ we have $E_{Q}=14.5 \mathrm{KeV}$. Similarly, a copper ion with charge $e Q$ has $N=34$ neutrons, $Z=29$ protons, and $(Z-Q)$ electrons. The mass number is

$$
A=N+Z=63
$$

and the relative atomic mass is $[2]$

$$
a=62.9295975(6) \text {. }
$$

For $Q=11$ the binding energy is $E_{Q}=1.29 \mathrm{KeV}$; for the fully stripped ion $(Q=29)$ we have $E_{Q}=45.0 \mathrm{KeV}[4,5]$.

\section{Kinetic Parameters}

In a circular accelerator the ion moves along an orbit of circumference $C$ with revolution frequency $f$. The radius of the orbit is defined to be $R=C /(2 \pi)$. The velocity of the ion is then

$$
v=2 \pi R f .
$$

This gives momentum, energy, and kinetic energy

$$
p=m c \beta \gamma, \quad E=m c^{2} \gamma, \quad W=m c^{2}(\gamma-1)
$$

where

$$
\beta=v / c, \quad \gamma=1 / \sqrt{1-\beta^{2}} .
$$

The magnetic rigidity of the ion in units of $\mathrm{Tm}$ is

$$
B \rho=k p / Q
$$

where $k=10^{9} / 299792458$ and $p$ is the momentum in units of $\mathrm{GeV} / \mathrm{c}$. The angular frequency is

$$
\omega=2 \pi f .
$$

We also define the phase-slip factor

$$
\eta=\frac{1}{\gamma_{t}^{2}}-\frac{1}{\gamma^{2}}
$$

where $\gamma_{t}$ is the transition gamma. 


\section{RF Parameters}

1. The stationary bucket area is

$$
A_{S}=8 \frac{R_{s}}{h c}\left\{\frac{2 e Q V_{g} E_{s}}{\pi h\left|\eta_{s}\right|}\right\}^{1 / 2}
$$

where $h$ is the RF harmonic number, $V_{g}$ is the total RF gap voltage per turn, and the subscript " $s$ " denotes parameter values for the synchronous particle.

2. The half-height of a bucket is

$$
\Delta E=\left(\frac{h \omega_{s}}{8 \sqrt{2}}\right) A_{S}\left|\left(\pi-2 \phi_{s}\right) \sin \phi_{s}-2 \cos \phi_{s}\right|^{1 / 2}
$$

where $\phi_{s}$ is the synchronous phase.

3. The synchronous phase is given by

$$
V_{g} \sin \phi_{s}=2 \pi R_{s} \rho_{s} \dot{B} / c
$$

where $\rho_{s}$ is the radius of curvature, $B$ is the magnetic field and $\dot{B}=d B / d t$. Employing Gaussian units $\left(R_{s}\right.$ and $\rho_{s}$ in $\mathrm{cm}$, $c=2.99792458 \times 10^{10} \mathrm{~cm} / \mathrm{s}$, and $\dot{B}$ in $\left.\mathrm{G} / \mathrm{s}\right)$ gives $V_{g} \sin \phi_{s}$ in Statvolts. Multiplying by 299.792458 then gives $V_{g} \sin \phi_{s}$ in Volts.

4. The width of a bucket is

$$
\Delta t=\frac{\left|\pi-\phi_{s}-\phi_{e}\right|}{h \omega_{s}}
$$

where the phase $\phi_{e}$ satisfies

$$
\cos \left(\pi-\phi_{s}\right)-\cos \phi_{e}=-\left(\pi-\phi_{s}-\phi_{e}\right) \sin \phi_{s} .
$$

5. The area of a bucket is

$$
A_{\mathrm{bk}}=\alpha\left(\phi_{s}\right) A_{S}
$$

where

$$
\alpha\left(\phi_{s}\right)=\frac{\sqrt{2}}{8} \int_{\phi_{L}}^{\phi_{R}}\left|\left(\pi-\phi_{s}-\phi\right) \sin \phi_{s}-\cos \phi_{s}-\cos \phi\right|^{1 / 2} d \phi .
$$


Below transition we have $\phi_{e}<\pi-\phi_{s}$ and the limits of integration are $\phi_{L}=\phi_{e}$ and $\phi_{R}=\pi-\phi_{s}$. Above transition we have $\pi-\phi_{s}<\phi_{e}$ and the limits of integration are $\phi_{L}=\pi-\phi_{s}$ and $\phi_{R}=\phi_{e}$. The integral $\alpha\left(\phi_{s}\right)$ must be evaluated numerically. An approximate expression is $[6]$

$$
\alpha\left(\phi_{s}\right) \approx \frac{1-\sin \phi_{s}}{1+\sin \phi_{s}}
$$

6. The synchrotron frequency for small-amplitude oscillations about $\phi_{s}$ is

$$
F_{s}=\frac{c}{2 \pi R_{s}}\left\{\frac{-h \eta_{s} e Q V_{g} \cos \phi_{s}}{2 \pi E_{s}}\right\}^{1 / 2}
$$

and the corresponding synchrotron tune is $Q_{s}=2 \pi F_{s} / \omega_{s}$. Note that measurement of $F_{s}$ gives a value for $V_{g} \cos \phi_{s}$, while measurement of $d B / d t$ gives a value for $V_{g} \sin \phi_{s}$. These two can be used to obtain $V_{g}$ and $\phi_{s}$.

7. Let $\phi_{l}$ and $\phi_{r}$ be the phases at the left and right boundries of a bunch matched to a bucket. We have

$$
\phi_{l}<\phi_{s}<\phi_{r}
$$

and the width of the bunch is

$$
\Delta t=\frac{\Delta \phi}{h \omega_{s}}, \quad \Delta \phi=\phi_{r}-\phi_{l} .
$$

In terms of $\Delta \phi$ and $\phi_{s}$ we have

$$
\phi_{r}=\frac{\Delta \phi}{2}+\arcsin \left\{\frac{\Delta \phi \sin \phi_{s}}{2 \sin (\Delta \phi / 2)}\right\}
$$

and

$$
\phi_{l}=-\frac{\Delta \phi}{2}+\arcsin \left\{\frac{\Delta \phi \sin \phi_{s}}{2 \sin (\Delta \phi / 2)}\right\} .
$$

If $\Delta \phi$ is small we have

$$
\sin (\Delta \phi / 2) \approx \frac{\Delta \phi}{2}, \quad \frac{\Delta \phi \sin \phi_{s}}{2 \sin (\Delta \phi / 2)} \approx \sin \phi_{s}
$$

and

$$
\phi_{l} \approx \phi_{s}-\frac{\Delta \phi}{2}, \quad \phi_{r} \approx \phi_{s}+\frac{\Delta \phi}{2} .
$$


8. The half-height of a bunch matched to a bucket is

$$
\Delta E=\left(\frac{h \omega_{s}}{8 \sqrt{2}}\right) A_{S}\left|\cos \phi_{r}-\cos \phi_{s}+\left(\phi_{r}-\phi_{s}\right) \sin \phi_{s}\right|^{1 / 2} .
$$

9. The area of a bunch matched to a bucket is

$$
A_{\mathrm{b}}=F\left(\phi_{s}, \Delta \phi\right) A_{S}
$$

where

$$
F\left(\phi_{s}, \Delta \phi\right)=\frac{\sqrt{2}}{8} \int_{\phi_{l}}^{\phi_{r}}\left|\cos \phi_{l}-\cos \phi+\left(\phi_{l}-\phi\right) \sin \phi_{s}\right|^{1 / 2} d \phi .
$$

The integral $F\left(\phi_{s}, \Delta \phi\right)$ must be evaluated numerically. If $\Delta \phi$ is small we have

$$
F\left(\phi_{s}, \Delta \phi\right) \approx \frac{\pi}{64}(\Delta \phi)^{2}\left|\cos \phi_{s}\right|^{1 / 2} .
$$

\section{Ring Parameters}

\begin{tabular}{|c|c|c|c|c|}
\hline Parameter & Booster & AGS & RHIC & Unit \\
\hline$C_{I}$ & $C_{b}$ & $C_{a}$ & $C_{r}+\delta C$ & $\mathrm{~m}$ \\
\hline$C_{E}$ & $C_{a} / 4$ & $4\left(C_{r}+\delta C\right) / 19$ & $C_{r}+\delta C$ & $\mathrm{~m}$ \\
\hline$\rho$ & 13.8656 & 85.378351 & 242.7806 & $\mathrm{~m}$ \\
\hline$\gamma_{\mathrm{tr}}$ & 4.832 & 8.5 & 22.89 & \\
\hline
\end{tabular}

Here $C_{I}$ and $C_{E}$ are the circumferences of the closed orbits in the machines at injection and extraction (or store) respectively. $C_{b}, C_{a}$, and $C_{r}$ are the circumferences of the "design" orbits in Booster, AGS, and RHIC respectively. These are

$$
C_{b}=201.780, \quad C_{a}=2 \pi(128.4526), \quad C_{r}=3833.845181
$$

meters. $\delta C$ is the shift (if any) of the RHIC orbit circumference from the design value $C_{r}$. Note that $4\left(C_{r} / 19\right)=2 \pi(128.4580) \mathrm{m}$ which gives an AGS radius at extraction approximately $5 \mathrm{~mm}$ larger than the "design" AGS radius $(128.4526 \mathrm{~m}$ ) reported by Bleser [7,8]. The radius of curvature $\rho$ in the Booster and AGS main dipoles is given in Refs. [7, 8, 9]. The RHIC ring parameters are taken from Ref. [10] and from MAD runs by Steve Tepikian. 
Copper and gold ions respectively are accelerated in the blue and yellow rings of RHIC. Because the ions have different magnetic rigidities and must pass through the same DX magnets, the orbit circumferences in the blue and yellow rings are not the same. Steve Tepikian has calculated the circumference shifts $\delta C$ in each ring at injection and at store. These are given in items 7 and 8 of the next section.

\section{$5 \quad$ Initial Conditions and Assumptions}

1. The revolution frequency of the Au32+ and Cu11+ ions (from EBIS) at Booster injection is $96.100 \mathrm{kHz}$.

2. The revolution frequency of the Au32+ ion at Booster extraction is $f=658.91 \mathrm{KHz}[11]$. The corresponding magnetic rigidity is $B \rho=9.4620277 \mathrm{Tm}$. The rigidity that can be extracted from Booster into the BTA line is limited by the F3 extraction kicker. The advertised limit is $B \rho=9.5 \mathrm{Tm}[12]$. We have successfully extracted Au31+ ions (originating from Tandem) at $B \rho=9.43 \mathrm{Tm}$ for several years.

3. The revolution frequency of the $\mathrm{Cu} 11+$ ion at Booster extraction is $f=660.15 \mathrm{KHz}$ [13]. The corresponding magnetic rigidity is $B \rho=8.8149099 \mathrm{Tm}$.

4. The set revolution frequency of the Au77+ ion at AGS injection is $f=163.125 \mathrm{KHz}$. This gives an energy loss of $2.453 \mathrm{MeV}$ per nucleon in the BTA stripper.

5. The set revolution frequency of the Cu29+ ion at AGS injection is $f=164.938 \mathrm{KHz}$. This gives an energy loss of $0.1539 \mathrm{MeV}$ per nucleon in the BTA stripper.

6. The magnetic rigidity of the Au79+ ion at RHIC injection is taken to be $B \rho=81.1137824 \mathrm{Tm}$. The revolution frequency of the $\mathrm{Cu} 29+$ ion at RHIC injection is the same as that of the Au79+ ion.

7. The orbit circumference for copper ions in the blue ring at injection is $0.611 \mathrm{~mm}$ greater than $C_{r}$. The orbit circumference for gold ions in the yellow ring at injection is $1.961 \mathrm{~mm}$ less than $C_{r}$.

8. The energy of the gold ion at RHIC Store is $100 \mathrm{GeV}$ per nucleon. The revolution frequency of the copper ion is the same as that of the 
gold ion. The orbit circumference for copper ions in the blue ring at store is $0.539 \mathrm{~mm}$ greater than $C_{r}$. The orbit circumference for gold ions in the yellow ring at store is $1.898 \mathrm{~mm}$ less than $C_{r}$.

The parameter values given in the following sections are calculated with these initial conditions and assumptions. For many of the parameters more digits are given than would be warranted by the precision with which the parameter could be measured; this is done for computational convenience.

\section{Inflector Voltage}

At Booster injection, the voltage $V_{I}$ required for particles with mass $m$, velocity $c \beta$, and charge $Q$ to follow the nominal trajectory through the inflector is given by

$$
e V_{I}=\frac{G}{R_{I}}\left(\frac{m c^{2}}{Q}\right) \beta^{2} \gamma
$$

Here $G=0.021 \mathrm{~m}$ is the gap between the cathode and septum of the inflector and $R_{I}=8.74123 \mathrm{~m}$ is the radius of curvature along the nominal trajectory. Using the calculated values of $\beta$ and $\gamma$ at Booster injection, we obtain $V_{I}=57.743$ and $53.669 \mathrm{kV}$ respectively for the Au32+ and Cu11+ ions from EBIS.

Because of an unresolved calibration error, the actual setpoint for the inflector voltage needs to be

$$
V_{I}(\text { setpoint })=1.034 V_{I}
$$

which gives

$$
V_{I}(\text { setpoint })=59.707 \mathrm{kV}
$$

for $\mathrm{Au} 32+$ ions and

$$
V_{I}(\text { setpoint })=55.493 \mathrm{kV}
$$

for $\mathrm{Cu} 11+$ ions. 


\section{Booster Injection Field}

The nominal magnetic field in the Booster dipoles at injection is

$$
B=(B \rho) / \rho
$$

where $B \rho$ is given by (11) and $\rho$ is the nominal radius of curvature. Using the calculated values of $B \rho$ we obtain $B=893.96$ and 830.87 Gauss respectively for the $\mathrm{Au} 32+$ and $\mathrm{Cu} 11+$ ions from EBIS.

The magnetic field is measured with a Hall probe and the Booster Gauss Clock. The Hall probe sits in the reference dipole and gives the value of the field at BT0. The Gauss Clock gives the change in field between BT0 and the time of measurement. The measured field is defined to be the field at BT0 plus the field change given by the Gauss Clock. For Au32+ ions from EBIS the measured field at injection is 891.6 Gauss.

\section{$8 \quad$ AGS Injection Field}

Similarly, the nominal magnetic field in the AGS dipoles at injection is $B=454.96$ and 391.26 Gauss for the Au77+ and Cu29+ ions respectively.

\section{BTA Stripper}

The stripper used to strip gold ions in the BTA (Booster-To-AGS) transfer line consists of a $6.45 \mathrm{mg} / \mathrm{cm}^{2}$ aluminum foil followed by a $8.39 \mathrm{mg} / \mathrm{cm}^{2}$ carbon foil. In Section 20 we use these surface densities to calculate the energy loss of Au77+ ions in the foils.

The stripper used to strip copper ions consists of a $4.38 \mathrm{mg} / \mathrm{cm}^{2}$ nickel foil followed by a $6.4 \mathrm{mg} / \mathrm{cm}^{2}$ aluminum foil. 


\section{AGS Injection Septum Magnet Current}

The field required in the L20 septum magnet is

$$
B=(B \rho) / \rho
$$

where $B \rho$ is the magnetic rigidity of the beam and $\rho=18.625 \mathrm{~m}[15]$ is the radius of curvature of the nominal trajectory through the magnet. The required current is given by

$$
N I=g B / \mu_{0}
$$

where $N=1$ is the number of conductor turns; $g=0.0467 \mathrm{~m}$ [15] is the magnet gap; and $\mu_{0}=4 \pi \times 10^{-7} \mathrm{Tm} / \mathrm{A}$.

For Au77+ ions at injection, the magnetic rigidity is $B \rho=3.88434088 \mathrm{Tm}$. This gives $B=0.208555 \mathrm{~T}$ and $I=7750 \mathrm{~A}$.

For $\mathrm{Cu} 29+$ ions at injection, the magnetic rigidity is $B \rho=3.3405536 \mathrm{Tm}$. This gives $B=0.179359 \mathrm{~T}$ and $I=6665 \mathrm{~A}$.

For comparison, the magnetic rigidity of polarized protons at AGS

injection is $B \rho=7.205178 \mathrm{Tm}$. This gives $B=0.3869 \mathrm{~T}$ and $I=14380 \mathrm{~A}$.

\section{AGS Injection Kicker Current}

The current required in the A5 kicker is $[14,15]$

$$
I=\frac{B \rho}{K} \sin \phi
$$

where

$$
K=1.8718 \times 10^{-5} \mathrm{Tm} / \mathrm{A}
$$

and

$$
\phi=3.35 \text { milliradians }
$$

is the desired kick angle. Using the calculated values of $B \rho$ at AGS injection we obtain currents of 695.2 and 597.9 A respectively for Au77+ and $\mathrm{Cu} 29+$ ions. The maximum available current is $1100 \mathrm{~A}$. 


\section{AGS Injection Kicker Short Pulse Waveforms}

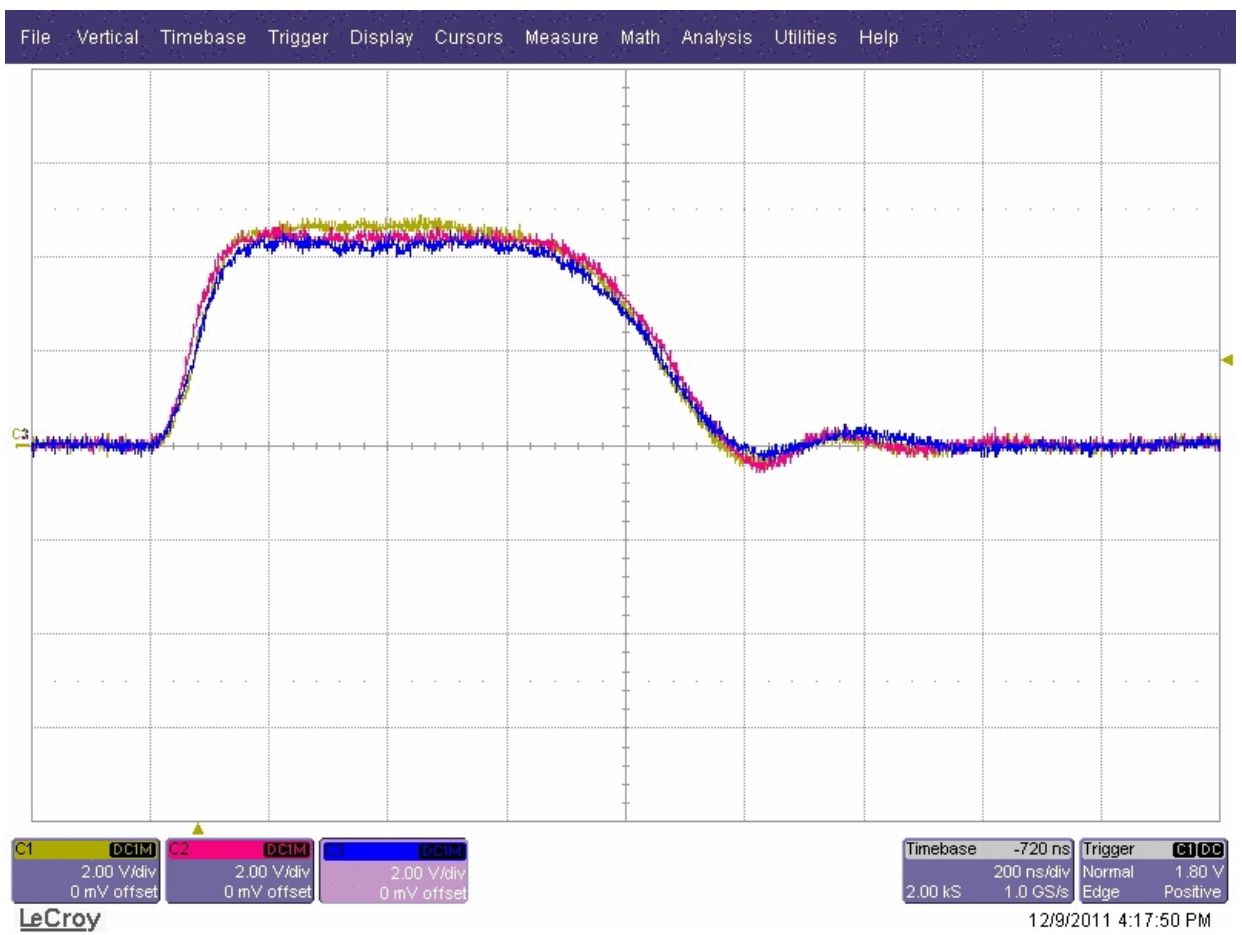

Figure 1: AGS injection kicker waveforms in the short pulse mode. The three traces are from the three modules of the kicker. They were taken by Yugang Tan on 9 Dec 2011. The time per division is 200 ns. The RF bucket width on the AGS injection porch is $383 \mathrm{~ns}$ for Au77+ ions and $379 \mathrm{~ns}$ for $\mathrm{Cu} 29+$ ions. In order to put beam into adjacent buckets, the rise time of the kicker must be less than or equal to the bucket width minus the bunch width. The rise time is approximately $100 \mathrm{~ns}$, which implies that the bunch width must be less than or equal to 283 and 279 ns for Au77+ and Cu29+ bunches respectively. A single bunch with these widths easily fits on the flattop portion of the pulse which is some $600 \mathrm{~ns}$ long. The total width of the pulse is some $1200 \mathrm{~ns}$. With this kicker pulse one could in principle fill 14 of the $16 \mathrm{RF}$ buckets on the AGS injection porch. The pulse is too wide to fill the remaining buckets without interfering with beam in the adjacent buckets. This was not an issue during the run as only 8 of the buckets needed to be filled. The filling pattern was four adjacent filled buckets followed by four adjacent empty buckets. 


\section{AGS Injection Kicker Long Pulse Waveforms}

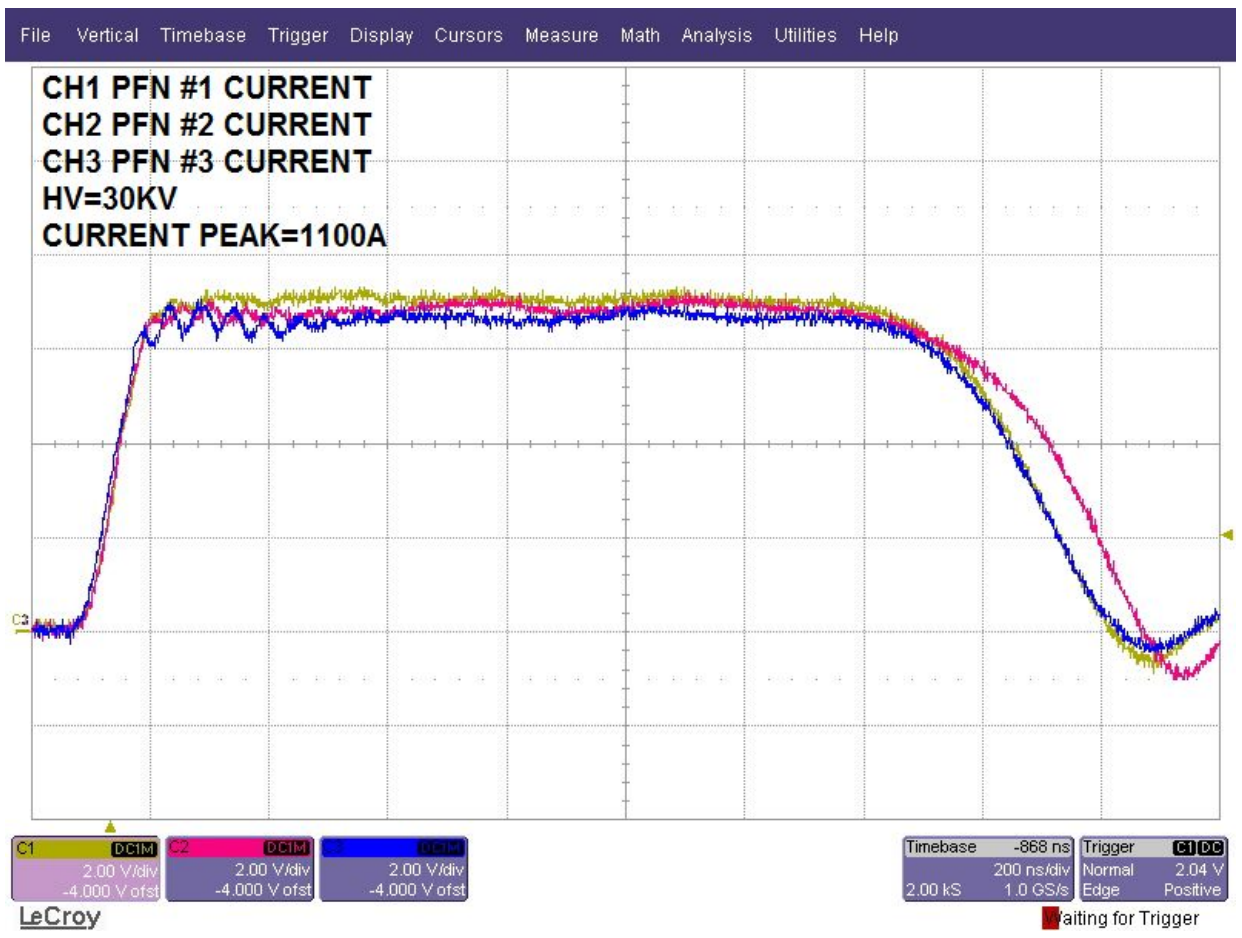

Figure 2: AGS injection kicker waveforms in the long pulse mode. The three traces are from the three modules of the kicker. They were taken by Yugang Tan in Oct 2010. The time per division is 200 ns. The RF bucket width on the AGS injection porch is 383 ns for Au77+ ions and 379 ns for Cu29+ ions. Here the flattop portion of the pulse is some $1300 \mathrm{~ns}$ long. The total pulse width is some 2000 ns. In principle this kicker pulse could be used to fill 8 buckets wtih a filling pattern of four adjacent filled buckets followed by four adjacent empty buckets. 


\section{Gold Parameters in Booster}

\begin{tabular}{|c|c|c|c|c|}
\hline Parameter & Injection & Merge porch & Extraction & Unit \\
\hline$Q$ & 32 & 32 & 32 & \\
\hline$m c^{2}$ & 183.456851 & 183.456851 & 183.456851 & $\mathrm{GeV}$ \\
\hline$W / A$ & 1.95418043 & 49.259795 & 107.75879 & $\mathrm{MeV}$ \\
\hline$c p / A$ & 60.3613437 & 306.87652 & 460.77475 & $\mathrm{MeV}$ \\
\hline$E / A$ & 0.933207234 & 0.98051285 & 1.0390118 & $\mathrm{GeV}$ \\
\hline$B \rho$ & 1.23952258 & 6.3017214 & 9.4620277 & $\mathrm{Tm}$ \\
\hline$\beta$ & 0.0646816072 & 0.31297552 & 0.44347401 & \\
\hline$\gamma-1$ & 0.00209844191 & 0.052896251 & 0.11571376 & \\
\hline$\eta$ & -0.953 & -0.859 & -0.7605 & \\
\hline$\epsilon_{H}(95 \%)$ & $12.0 \pi$ & $12.0 \pi$ & $12.0 \pi$ & $\mathrm{mm} \mathrm{mrad}$ \\
\hline$\epsilon_{V}(95 \%)$ & $5.64 \pi$ & $5.64 \pi$ & $5.64 \pi$ & $\mathrm{mm} \mathrm{mrad}$ \\
\hline$h$ & 4 & 1 & 1 & \\
\hline$h f$ & 384.400 & 465.000 & 658.910 & $\mathrm{KHz}$ \\
\hline$R$ & $201.780 /(2 \pi)$ & $201.780 /(2 \pi)$ & $128.4526 / 4$ & $\mathrm{~m}$ \\
\hline
\end{tabular}

Here $\epsilon_{H}$ and $\epsilon_{V}$ are the normalized horizontal and vertical transverse emittances. These follow from the assumption that during injection the horizontal and vertical acceptances in Booster are completely filled. The horizontal and vertical acceptances are $185 \pi$ and $87 \pi \mathrm{mm} \mathrm{mrad}$ (un-normalized) respectively.

\begin{tabular}{|c|c|c|c|c|c|}
\hline Parameter & Injection & Extraction & Extraction & Extraction & Unit \\
\hline$V_{g}$ & 0.5 & 30 & 30 & 30 & $\mathrm{kV}$ \\
$A_{S}$ & 4.7485 & 347.55 & 347.55 & 347.55 & $\mathrm{eV} \mathrm{s}$ \\
$d B / d t$ & 0 & $\mathbf{7 0}$ & $\mathbf{3 5}$ & $\mathbf{0}$ & $\mathrm{G} / \mathrm{ms}$ \\
\hline$\phi_{s}$ & 0 & 40.753 & 19.050 & 0 & degrees \\
$F_{s}$ & 0.3414 & 0.9743 & 1.0884 & 1.1195 & $\mathrm{kHz}$ \\
$A_{\mathrm{bk}}$ & 4.7485 & 70.783 & 176.91 & 347.55 & $\mathrm{eV} \mathrm{s}$ \\
\hline$A_{b}$ & 4.728 & 23.64 & 23.64 & 23.64 & $\mathrm{eV} \mathrm{s}$ \\
$\Delta t$ & 2533 & 313.0 & 291.7 & 287.0 & $\mathrm{~ns}$ \\
$\Delta E$ & 1.432 & 48.63 & 51.83 & 52.64 & $\mathrm{MeV}$ \\
\hline
\end{tabular}

\begin{tabular}{|c|c|c|c|}
\hline Parameter & Injection & Extraction & Unit \\
\hline No. of Bunches & 4 & 1 & \\
\hline Bucket Width & 2601.4568 & 1517.65795 & $\mathrm{~ns}$ \\
\hline Ions/Bunch & $1.25 / 4$ & 0.981 & $10^{9}[16]$ \\
\hline Bunch Area & $0.096 / 4$ & 0.12 & $\mathrm{eV} \mathrm{s} / A$ \\
\hline
\end{tabular}




\section{Copper Parameters in Booster}

\begin{tabular}{|c|c|c|c|c|}
\hline Parameter & Injection & Merge Porch & Extraction & Unit \\
\hline$Q$ & 11 & 11 & 11 & \\
\hline$m c^{2}$ & 58.6129266 & 58.6129266 & 58.6129266 & $\mathrm{GeV}$ \\
\hline$W / A$ & 1.952314629 & 49.212763 & 108.134927 & $\mathrm{MeV}$ \\
\hline$c p / A$ & 60.3037121 & 306.58352 & 461.41395 & $\mathrm{MeV}$ \\
\hline$E / A$ & 0.9323162295 & 0.97957668 & 1.0384988 & $\mathrm{GeV}$ \\
\hline$B \rho$ & 1.15204968 & 5.8570100 & 8.8149099 & $\mathrm{Tm}$ \\
\hline$\beta$ & 0.06468160717 & 0.31295772 & 0.44430858 & \\
\hline$\gamma-1$ & 0.00209844191 & 0.052896251 & 0.116228634 & \\
\hline$\eta$ & -0.953 & -0.859 & -0.760 & \\
\hline$\epsilon_{H}(95 \%)$ & $12.0 \pi$ & $12.0 \pi$ & $12.0 \pi$ & $\mathrm{mm} \mathrm{mrad}$ \\
\hline$\epsilon_{V}(95 \%)$ & $5.64 \pi$ & $5.64 \pi$ & $5.64 \pi$ & $\mathrm{mm} \mathrm{mrad}$ \\
\hline$h$ & 4 & 1 & 1 & \\
\hline$h f$ & 0.384400 & 465.000 & 660.15 & $\mathrm{KHz}$ \\
\hline$R$ & $201.780 /(2 \pi)$ & $201.780 /(2 \pi)$ & $128.4526 / 4$ & $\mathrm{~m}$ \\
\hline
\end{tabular}

Here $\epsilon_{H}$ and $\epsilon_{V}$ are the normalized horizontal and vertical transverse emittances. These follow from the assumption that during injection the horizontal and vertical acceptances in Booster are completely filled. The horizontal and vertical acceptances are $185 \pi$ and $87 \pi \mathrm{mm} \mathrm{mrad}$ (un-normalized) respectively.

\begin{tabular}{|c|c|c|c|c|c|}
\hline Parameter & Injection & Extraction & Extraction & Extraction & Unit \\
\hline$V_{g}$ & 0.500 & 30 & 30 & 30 & $\mathrm{kV}$ \\
$A_{S}$ & 1.5736 & 115.26 & 115.26 & 115.26 & $\mathrm{eV} \mathrm{s}$ \\
$d B / d t$ & 0 & $\mathbf{7 0}$ & $\mathbf{3 5}$ & $\mathbf{0}$ & $\mathrm{G} / \mathrm{ms}$ \\
\hline$\phi_{s}$ & 0 & 40.753 & 19.050 & 0 & degrees \\
$F_{s}$ & 0.3541 & 1.010 & 1.128 & 1.160 & $\mathrm{kHz}$ \\
$A_{\mathrm{bk}}$ & 1.5736 & 23.47 & 58.67 & 115.26 & $\mathrm{eV} \mathrm{s}$ \\
\hline$A_{b}$ & 1.512 & 7.56 & 7.56 & 7.56 & $\mathrm{eV} \mathrm{s}$ \\
$\Delta t$ & 2364 & 306.5 & 285.8 & 281.2 & $\mathrm{~ns}$ \\
$\Delta E$ & 0.4702 & 15.88 & 16.92 & 17.18 & $\mathrm{MeV}$ \\
\hline
\end{tabular}

\begin{tabular}{|c|c|c|c|}
\hline Parameter & Injection & Extraction & Unit \\
\hline No. of Bunches & 4 & 1 & \\
\hline Bucket Width & 2601.4568 & 1514.8072 & $\mathrm{~ns}$ \\
\hline Ions/Bunch & $4.94 / 4$ & 4.06 & $10^{9}[17]$ \\
\hline Bunch Area & $0.096 / 4$ & 0.12 & $\mathrm{eV} \mathrm{s} / A$ \\
\hline
\end{tabular}




\section{Gold Parameters in AGS}

\begin{tabular}{|c|c|c|c|c|}
\hline Parameter & Injection & Transition & Extraction & Unit \\
\hline$Q$ & 77 & 77 & 77 & \\
\hline$m c^{2}$ & 183.434174 & 183.434174 & 183.434174 & $\mathrm{GeV}$ \\
\hline$W / A$ & 0.10529199 & 6.98353456 & 8.86486832 & $\mathrm{GeV}$ \\
\hline$c p / A$ & 0.45515837 & 7.85970883 & 9.75165221 & $\mathrm{GeV}$ \\
\hline$E / A$ & 1.0364299 & 7.91467250 & 9.79600627 & $\mathrm{GeV}$ \\
\hline$B \rho$ & 3.88434088 & 67.0750887 & 83.2210138 & $\mathrm{Tm}$ \\
\hline$\beta$ & 0.43915981 & 0.993055472 & 0.995472231 & \\
\hline$\gamma$ & 1.1130788 & 8.5000 & 10.5204673 & \\
\hline$\eta$ & -0.793 & 0.0 & 0.00481 & \\
\hline$\epsilon_{H}(95 \%)$ & $\leq 12 \pi$ & $\leq 12 \pi$ & $\leq 12 \pi$ & $\mathrm{mm} \mathrm{mrad}$ \\
\hline$\epsilon_{V}(95 \%)$ & $\leq 12 \pi$ & $\leq 12 \pi$ & $\leq 12 \pi$ & $\mathrm{mm} \mathrm{mrad}$ \\
\hline$h$ & 16 & 12 & 12 & \\
\hline$h f$ & 2.610000 & 4.42642072 & 4.43700951 & $\mathrm{MHz}$ \\
\hline$R$ & 128.4526 & 128.4526 & 128.45798 & $\mathrm{~m}$ \\
\hline
\end{tabular}

\begin{tabular}{|c|c|c|c|c|}
\hline Parameter & Injection & Injection & Extraction & Unit \\
\hline$h$ & 16 & 4 & 12 & \\
\hline$V_{g}$ & 33.84 & 22 & 161.9 & $\mathrm{kV}$ \\
$A_{S}$ & 35.00 & 225.7 & 4655 & $\mathrm{eV} \mathrm{s}$ \\
$d B / d t$ & 0 & 0 & 0 & $\mathrm{G} / \mathrm{ms}$ \\
\hline$\phi_{s}$ & 0 & 0 & 180 & degrees \\
$F_{s}$ & 1.886 & 0.7603 & 0.0904 & $\mathrm{kHz}$ \\
$A_{\mathrm{bk}}$ & 35.00 & 225.7 & 4655 & $\mathrm{eV} \mathrm{s}$ \\
\hline$A_{b}$ & 27.58 & 110.3 & 110.3 & $\mathrm{eV} \mathrm{s}$ \\
$\Delta t$ & 287.0 & 833.9 & 25 & $\mathrm{~ns}[18]$ \\
$\Delta E$ & 66.24 & 87.27 & 2812 & $\mathrm{MeV}$ \\
\hline
\end{tabular}

\begin{tabular}{|c|c|c|c|c|}
\hline Parameter & Injection & Injection & Extraction & Unit \\
\hline$h$ & 16 & 4 & 12 & \\
\hline Bucket Width & 383.142 & 1532.567 & 225.377 & ns \\
\hline No. of Bunches & 8 & 2 & 2 & \\
\hline Ions/Bunch & 0.556 & 2.224 & 1.62 & $10^{9}[16]$ \\
\hline Bunch Area & 0.14 & 0.56 & 0.56 & $\begin{array}{c}\mathrm{eV} \mathrm{s} / A \\
{[19,20]}\end{array}$ \\
\hline
\end{tabular}




\section{Copper Parameters in AGS}

\begin{tabular}{|c|c|c|c|c|}
\hline Parameter & Injection & Transition & Extraction & Unit \\
\hline$Q$ & 29 & 29 & 29 & \\
\hline$m c^{2}$ & 58.60377233 & 58.60377233 & 58.60377233 & $\mathrm{GeV}$ \\
\hline$W / A$ & 0.1079641423 & 6.97663957 & 8.85683601 & $\mathrm{GeV}$ \\
\hline$c p / A$ & 0.4609954061 & 7.85194877 & 9.74274763 & $\mathrm{GeV}$ \\
\hline$E / A$ & 1.0381827506 & 7.90685818 & 9.78705462 & $\mathrm{GeV}$ \\
\hline$B \rho$ & 3.340553613 & 56.8983020 & 70.5997725 & $\mathrm{Tm}$ \\
\hline$\beta$ & 0.44404071036 & 0.993055472 & 0.995472899 & \\
\hline$\gamma$ & 1.1160631935 & 8.5000 & 10.5212415 & \\
\hline$\eta$ & -0.789 & 0.0 & 0.00481 & \\
\hline$\epsilon_{H}(95 \%)$ & $\leq 12 \pi$ & $\leq 12 \pi$ & $\leq 12 \pi$ & $\mathrm{mm} \mathrm{mrad}$ \\
\hline$\epsilon_{V}(95 \%)$ & $\leq 12 \pi$ & $\leq 12 \pi$ & $\leq 12 \pi$ & $\mathrm{mm} \mathrm{mrad}$ \\
\hline$h$ & 16 & 12 & 12 & \\
\hline$h f$ & 2.639008 & 4.42642072 & 4.43700951 & $\mathrm{MHz}$ \\
\hline$R$ & 128.4526 & 128.4526 & 128.45800 & $\mathrm{~m}$ \\
\hline
\end{tabular}

\begin{tabular}{|c|c|c|c|c|}
\hline Parameter & Injection & Injection & Extraction & Unit \\
\hline$h$ & 16 & 4 & 12 & \\
\hline$V_{g}$ & 29.22 & 22 & 137.7 & $\mathrm{kV}$ \\
$A_{S}$ & 11.33 & 78.62 & 1489 & $\mathrm{eV} \mathrm{s}$ \\
$d B / d t$ & 0 & 0 & 0 & $\mathrm{G} / \mathrm{ms}$ \\
\hline$\phi_{s}$ & 0 & 0 & 180 & degrees \\
$F_{s}$ & 1.895 & 0.8222 & 0.09057 & $\mathrm{kHz}$ \\
$A_{\mathrm{bk}}$ & 11.33 & 78.62 & 1489 & $\mathrm{eV} \mathrm{s}$ \\
\hline$A_{b}$ & 8.82 & 35.28 & 35.28 & $\mathrm{eV} \mathrm{s}$ \\
$\Delta t$ & 281.2 & 783.9 & 25 & $\mathrm{~ns}[21]$ \\
$\Delta E$ & 21.6 & 29.6 & 900 & $\mathrm{MeV}$ \\
\hline
\end{tabular}

\begin{tabular}{|c|c|c|c|c|}
\hline Parameter & Injection & Injection & Extraction & Unit \\
\hline$h$ & 16 & 4 & 12 & \\
\hline Bucket Width & 378.930 & 1515.721 & 225.377 & $\mathrm{~ns}$ \\
\hline No. of Bunches & 8 & 2 & 2 & \\
\hline Ions/Bunch & 2.8 & 11.2 & 6.5 & $10^{9}[17]$ \\
\hline Bunch Area & 0.14 & 0.56 & 0.56 & $\begin{array}{c}\mathrm{eV} \mathrm{s} / N \\
{[22]}\end{array}$ \\
\hline
\end{tabular}




\section{Gold Parameters in RHIC}

\begin{tabular}{|c|c|c|c|c|}
\hline Parameter & Injection & Transition & Store & Unit \\
\hline$Q$ & 79 & 79 & 79 & \\
\hline$m c^{2}$ & 183.433337 & 183.433337 & 183.433337 & $\mathrm{GeV}$ \\
\hline$W / A$ & 8.86482786 & 20.3825165 & 99.0688663 & $\mathrm{GeV}$ \\
\hline$c p / A$ & 9.75160770 & 21.2933012 & 99.9956649 & $\mathrm{GeV}$ \\
\hline$E / A$ & 9.79596155 & 21.3136502 & 100.000000 & $\mathrm{GeV}$ \\
\hline$B \rho$ & 81.1137824 & 177.117482 & 831.763013 & $\mathrm{Tm}$ \\
\hline$\beta$ & 0.995472231 & 0.999045259 & 999956649 & \\
\hline$\gamma$ & 10.5204673 & 22.8900 & 107.395964 & \\
\hline$\eta$ & -0.00713 & 0.0 & 0.00182 & \\
\hline$\epsilon_{H}(95 \%)$ & $\leq 10 \pi$ & $\leq 10 \pi$ & $\leq 10 \pi$ & $\mathrm{mm} \mathrm{mrad}$ \\
\hline$\epsilon_{V}(95 \%)$ & $\leq 10 \pi$ & $\leq 10 \pi$ & $\leq 10 \pi$ & $\mathrm{mm} \mathrm{mrad}$ \\
\hline$h$ & 360 & 360 & 2520 & \\
\hline$h f$ & 28.0232179485 & 28.1238011 & 197.046198131 & $\mathrm{MHz}$ \\
\hline$\delta C$ & -1.961 & -1.961 & -1.898 & $\mathrm{~mm}$ \\
\hline
\end{tabular}

\begin{tabular}{|c|c|c|c|}
\hline Parameter & Injection & Store & Unit \\
\hline$h$ & 360 & 2520 & \\
\hline$V_{g}$ & 302.5 & 3000 & $\mathrm{kV}$ \\
$A_{S}$ & 153.0 & 164.4 & $\mathrm{eV} \mathrm{s}$ \\
$d B / d t$ & 0 & 0 & $\mathrm{G} / \mathrm{ms}$ \\
\hline$\phi_{s}$ & 0 & 180 & degrees \\
$F_{s}$ & 0.176 & 0.232 & $\mathrm{kHz}$ \\
$A_{\mathrm{bk}}$ & 153.0 & 164.4 & $\mathrm{eV} \mathrm{s}$ \\
$A_{b}$ & 110.32 & 110.32 & $\mathrm{eV} \mathrm{s}$ \\
\hline$A_{b}$ & 0.56 & 0.56 & $\mathrm{eV} \mathrm{s} / \mathrm{A}[23]$ \\
$\Delta t$ & 25 & 3.38 & $\mathrm{~ns}$ \\
$\Delta E$ & 3002 & 22022 & $\mathrm{MeV}$ \\
\hline
\end{tabular}




\section{Copper Parameters in RHIC}

\begin{tabular}{|c|c|c|c|c|}
\hline Parameter & Injection & Transition & Store & Unit \\
\hline$Q$ & 29 & 29 & 29 & \\
\hline$m c^{2}$ & 58.60377233 & 58.60377233 & 58.60377233 & $\mathrm{GeV}$ \\
\hline$W / A$ & 8.85683601 & 20.3624853 & 99.7120297 & $\mathrm{GeV}$ \\
\hline$c p / A$ & 9.74274764 & 21.2723749 & 1.006379493 & $\mathrm{GeV}$ \\
\hline$E / A$ & 9.78705462 & 21.29270394 & 100.6422483 & $\mathrm{GeV}$ \\
\hline$B \rho$ & 70.5997725 & 154.147976 & 729.262072 & $\mathrm{Tm}$ \\
\hline$\beta$ & 0.995472899 & 0.99904526 & 0.9999572842 & \\
\hline$\gamma$ & 10.5212415 & 22.8900 & 108.1920394 & \\
\hline$\eta$ & -0.00713 & 0.0 & 0.00182 & \\
\hline$\epsilon_{H}(95 \%)$ & $\leq 10 \pi$ & $\leq 10 \pi$ & $\leq 10 \pi$ & $\mathrm{mm} \mathrm{mrad}$ \\
\hline$\epsilon_{V}(95 \%)$ & $\leq 10 \pi$ & $\leq 10 \pi$ & $\leq 10 \pi$ & $\mathrm{mm} \mathrm{mrad}$ \\
\hline$h$ & 360 & 360 & 2520 & \\
\hline$h f$ & 28.0232179485 & 28.1237822 & 197.046198131 & $\mathrm{MHz}$ \\
\hline$\delta C$ & 0.611 & 0.611 & 0.539 & $\mathrm{~mm}$ \\
\hline
\end{tabular}

\begin{tabular}{|c|c|c|c|}
\hline Parameter & Injection & Store & Unit \\
\hline$h$ & 360 & 2520 & \\
\hline$V_{g}$ & 263.7 & 3000 & $\mathrm{kV}$ \\
$A_{S}$ & 48.93 & 56.49 & $\mathrm{eV} \mathrm{s}$ \\
$d B / d t$ & 0 & 0 & $\mathrm{G} / \mathrm{ms}$ \\
\hline$\phi_{s}$ & 0 & 180 & degrees \\
$F_{s}$ & 0.176 & 0.248 & $\mathrm{kHz}$ \\
$A_{\mathrm{bk}}$ & 48.93 & 56.49 & $\mathrm{eV} \mathrm{s}$ \\
$A_{b}$ & 35.28 & 35.28 & $\mathrm{eV} \mathrm{s}$ \\
\hline$A_{b}$ & 0.56 & 0.56 & $\mathrm{eV} \mathrm{s} / \mathrm{A}[23]$ \\
$\Delta t$ & 25 & 3.22 & $\mathrm{~ns}$ \\
$\Delta E$ & 960 & 7342 & $\mathrm{MeV}$ \\
\hline
\end{tabular}




\section{Au77+ Energy Loss in the BTA Stripper Foils}

The stripper used to strip gold ions consists of a $6.45 \mathrm{mg} / \mathrm{cm}^{2}$ aluminum foil followed by a $8.39 \mathrm{mg} / \mathrm{cm}^{2}$ "glassy" carbon foil [24, 25]. We can estimate the energy loss in the foils as follows:

The kinetic energy of a proton that has the same velocity as the Au77+ ion just upstream of the aluminum foil is

$$
W_{p}=108.6 \mathrm{MeV} .
$$

The rate of energy loss of a proton passing through the foil with kinetic energy $W_{p}$ is $[26]$

$$
-\frac{d E_{p}}{d x}=5.348 \mathrm{MeV} \mathrm{cm}^{2} / \mathrm{g} .
$$

The rate of energy loss of the Au77+ ion is obtained by scaling the Bethe-Bloch result for protons [27]. Thus

$$
-\frac{d E}{d x}=-Z^{2} \frac{d E_{p}}{d x} \mathrm{~cm}^{2} / \mathrm{g}
$$

where $Z=77$. Multiplying this by the surface density of the aluminum foil $\left(6.45 \mathrm{mg} / \mathrm{cm}^{2}\right)$ gives

$$
\Delta E_{a}=1.038 \mathrm{MeV} \text { per nucleon. }
$$

This is the energy lost by the Au77+ ion upon passing through the aluminium foil. The kinetic energy of a proton that has the same velocity as the Au77+ ion just downstream of the aluminum foil is then

$$
W_{p}=107.5 \mathrm{MeV} \text {. }
$$

The rate of energy loss of a proton passing through the carbon foil with this kinetic energy is [26]

$$
-\frac{d E_{p}}{d x}=6.180 \mathrm{MeV} \mathrm{cm}^{2} / \mathrm{g} .
$$

Using this result in (46) with $Z=77$, and multiplying by the surface density of the carbon foil $\left(8.39 \mathrm{mg} / \mathrm{cm}^{2}\right)$ gives

$$
\Delta E_{c}=1.561 \mathrm{MeV} \text { per nucleon. }
$$

The total energy lost upon passing through both foils is then

$$
\Delta E=\Delta E_{a}+\Delta E_{c}=2.599 \mathrm{MeV} \text { per nucleon. }
$$

This agrees reasonably well with the value $2.453 \mathrm{MeV}$ per nucleon obtained in Section 5. 


\section{References}

[1] C.J. Gardner, "Simulations of Merging and Squeezing Bunches in Booster and AGS", C-A/AP/Note 460, July 2012.

[2] J.S. Coursey, D.J. Schwab, and R.A. Dragoset, "Atomic Weights and Isotopic Compositions", Nuclear Physics Data, Physical Reference Data, www.nist.gov.

[3] P.J. Mohr and B.N. Taylor, "Values of Fundamental Physical Constants", Physical Constants, Physical Reference Data, www.nist.gov.

[4] K.A. Brown, C. Gardner and P. Thieberger, "Rest Mass of Fully Stripped Ions in RHIC: Updated Values", C-A/AP/Note 293, October 2007.

[5] G.C. Rodrigues, P. Indelicato, J.P. Santos, P. Patte, and F. Parente, "Systematic Calculation of Total Atomic Energies of Ground State Configurations", Atomic Data and Nuclear Data Tables 86 (2004) $117-233$.

[6] S.Y. Lee, "Accelerator Physics", World Scientific, 1999, pp. 229-230

[7] E.J. Bleser, "Where are the AGS Magnets", Accelerator Division Technical Note 215, May 20, 1985.

[8] C.J. Gardner, "Notes on Orbit Equations in the AGS", C-A/AP/Note 164, September 2004.

[9] R. Thern, "Booster Dipole Production Measurements", Booster Technical Note 190, March 13, 1991.

[10] W. Fischer and S. Peggs, "RHIC Parameters", Revision of 3/18/97.

[11] K.L. Zeno, Booster-AGS-EBIS-2012 elog, 16 May 2012, entry 15:50.

[12] W. Zhang, R. Sanders, A. Soukas and J. Tuozzolo, "An Overview of the Fast Injection-Extraction Kicker Systems of the Brookhaven AGS-Booster Complex", PAC99, pp. 1264-1266.

[13] K.L. Zeno, Booster-AGS-EBIS-2012 elog, 22 May 2012, entry 16:56.

[14] C.J. Gardner, "Determination of the AGS Injection Kicker Strength from Beam Measurements", C-A/AP/Note 91, December 2002. 
[15] C.J. Gardner, "AGS Injection with an Additional Kicker in the A10 Straight Section", C-A/AP/Note 217, September 2005.

[16] K.L. Zeno, Booster-AGS-EBIS-2012 elog, 19 June 2012, entry 14:47.

[17] K.L. Zeno, Booster-AGS-EBIS-2012 elog, 19 June 2012, entry 19:43.

[18] K.L. Zeno, Booster-AGS-EBIS-2012 elog, 19 June 2012, entry 14:49.

[19] K.L. Zeno, Booster-AGS-EBIS-2012 elog, 19 June 2012, entry 15:11.

[20] K.L. Zeno, Booster-AGS-EBIS-2012 elog, 19 June 2012, entry 15:30.

[21] K.L. Zeno, Booster-AGS-EBIS-2012 elog, 6 June 2012, entry 18:21.

[22] K.L. Zeno, Booster-AGS-EBIS-2012 elog, 19 June 2012, entry 12:57.

[23] Here we take the longitudinal emittance to be the same as that at AGS extraction. This gives a lower bound on the longitudinal emittance in RHIC.

[24] C.J. Gardner, et al, "Setup and Performance of the RHIC Injector Accelerators for the 2007 Run with Gold Ions", Proceedings of PAC07, pp. 1862-1864.

[25] P. Thieberger, et al, "Improved Gold Ion Stripping at 0.1 and 10 $\mathrm{GeV} /$ nucleon for the Relativistic Heavy Ion Collider", Phys. Rev. ST Accelerators and Beams 11, 011001 (2008).

[26] M.J. Berger, J.S. Coursey, M.A. Zucker and J. Chang, "Stopping-Power and Range Tables for Electrons, Protons, and Helium Ions", www.nist.gov/physlab/data/star/index.cfm

[27] W.R. Leo, "Techniques for Nuclear and Particle Physics Experiments", Second Revised Edition, Springer-Verlag, 1994, pp. 24-28. 